\title{
Drug resistant gut bacteria mimic a host mechanism for anticancer drug clearance
}

Peter Spanogiannopoulos ${ }^{1}$, Patrick H. Bradley ${ }^{2,3}$, Jonathan Melamed ${ }^{4}$, Ysabella Noelle Amora Malig ${ }^{4}$, Kathy N. Lam ${ }^{1}$, Roy R. Gerona ${ }^{4}$, Katherine S. Pollard ${ }^{2,3,5}$, and Peter J. Turnbaugh ${ }^{1,5^{*}}$

${ }^{1}$ Department of Microbiology \& Immunology, University of California San Francisco, San Francisco, CA 94143, USA

${ }^{2}$ Gladstone Institutes, San Francisco, CA, USA

${ }^{3}$ Division of Biostatistics, Institute for Human Genetics, and Institute for Computational Health Sciences, University of California, San Francisco, CA, USA.

${ }^{4}$ Clinical Toxicology and Environmental Biomonitoring Laboratory, University of California, San Francisco, CA, 94143, USA

${ }^{5}$ Chan Zuckerberg Biohub, San Francisco, CA 94158, USA

*Correspondence to:

Peter J. Turnbaugh

Associate Professor

Department of Microbiology \& Immunology

513 Parnassus Avenue HSW 1529

San Francisco, CA 94143-0552

Peter.Turnbaugh@ucsf.edu

Office: (415) 502-3237

Fax: (415) 476-6185 
Microbiome surveys indicate that pharmaceuticals are the top predictor of inter-individual variations in gut microbial community structure ${ }^{1}$, consistent with in vitro evidence that nonantibiotic (i.e. host-targeted) drugs inhibit gut bacterial growth ${ }^{2}$ and are subject to extensive metabolism by the gut microbiome $e^{3,4}$. In oncology, bacterial metabolism has been implicated in both drug efficacy ${ }^{5,6}$ and toxicity ${ }^{7,8}$; however, the degree to which bacterial sensitivity and metabolism can be driven by conserved pathways also found in mammalian cells remains poorly understood. Here, we show that anticancer fluoropyrimidine drugs broadly inhibit the growth of diverse gut bacterial strains. Media supplementation, transcriptional profiling (RNA-seq), and bacterial genetics implicated pyrimidine metabolism as a key target in bacteria, as in mammalian cells. Drug resistant bacteria metabolized 5FU to its inactive metabolite dihydrofluorouracil (DHFU) mimicking the major host pathway for drug clearance. Functional orthologs of the bacterial operon responsible (preTA) are widespread across human gut bacteria from the Firmicutes and Proteobacteria phyla. The observed conservation of both the targets and pathways for metabolism of therapeutics across domains highlights the need to distinguish the relative contributions of human and microbial cells to drug disposition ${ }^{9}$, efficacy, and side effect profiles.

Although enormous advances in anticancer drug therapy have been made in the last decade, including the development of targeted therapies and immunotherapy, chemotherapeutic drugs, such as fluoropyrimidines, remain mainstay agents in the treatment of cancer ${ }^{10}$. Multiple lines of evidence support the scientific premise that fluoropyrimidines have extensive interactions with the human gut microbiome that may have downstream consequences for treatment outcomes. The oral fluoropyrimidine capecitabine (CAP), which is extensively metabolized to 5-fluorouracil (5FU), 5-fluorodeoxyuridine (5FdUR), and numerous other metabolites (Fig. 1a, Extended Data Fig. 1), meets the FDA criteria for a "highly variable" drug, with a coefficient of variation $>30 \%$ in intra-subject pharmacokinetic parameters combined with extensive variation between subjects ${ }^{11}$, which cannot be explained by the known dietary or host genetic risk factors ${ }^{12,13}$. Adverse reactions to CAP require dose adjustments in $\sim 35 \%$ of patients and complete discontinuation of therapy in $\sim 10 \%$ of patients; GI side effects are common ${ }^{14,15}$. Of note, fluoropyrimidines were designed to target conserved pathways essential for gut bacterial growth ${ }^{16,17}$ and recent evidence in rats has shown that these drugs alter the gut microbiota ${ }^{18-20}$. Consistent with these findings, cross-sectional analyses of the human gut microbiome have linked the use of anticancer drugs (including fluoropyrimidines) to changes in gut microbial community structure and function ${ }^{21-24}$. There is also evidence that the bacterial metabolism of fluoropyrimidines can have downstream consequences for host physiology; two recent studies in worms linked genetic differences in their bacterial food source (Escherichia coli) to sensitivity to $5 \mathrm{FU}^{6,25}$. Furthermore, the increasing administration of oral 
fluoropyrimidines increases the potential for interactions with the gut microbiota prior to first pass metabolism and absorption into general circulation.

These observations motivated us to conduct a systematic screen for human gut bacterial sensitivity to three fluoropyrimidine drugs: CAP, 5FU, and 5FdUR (Fig. 1a). We selected 47 sequenced and publicly available bacterial strains, spanning the 6 dominant phyla found in the human gut (Supplementary Table 1). On average, these bacterial species represent $46.0 \pm 19.7 \%$ of the gut microbiota $^{26}$. Drug sensitivity was evaluated by determining the minimal inhibitory concentration (MIC) in a rich medium that supported the growth of our entire strain collection (Supplementary Table 1). This screen revealed extensive variation in sensitivity to 5FU and 5FdUR (Fid. 1b), spanning >3 orders of magnitude ( $\min =100 \mathrm{ng} / \mathrm{ml}$; $\max >1 \mathrm{mg} / \mathrm{ml}$ ) (Supplementary Table 1). Interestingly, 13 (5FU) and 15 (5FdUR) bacterial strains were resistant to the highest concentration assayed, although 11 (5FU) and 8 (5FdUR) displayed partial growth inhibition (range: $12-92 \%$ of the growth control). Variation in sensitivity to 5FU and 5FdUR was significantly correlated (rho=0.475, $p$-value=0.0008; Extended Data Fig. 2a), as expected based on their shared substructure (Fig. 1a) and downstream metabolites (Extended Data Fig. 1). Bacterial phylogeny was a poor predictor of drug sensitivity with multiple examples of nearest neighbors with opposite phenotypes; for example, Parabacteroides distasonis (5FU MIC=300 ng/ml) and P. merdae (5FU MIC>1mg/ml). This variation was also apparent when grouping bacteria at higher taxonomic levels (Extended Data Fig. 2b). Based on this observation, we hypothesized that bacteria may be able to rapidly evolve resistance to fluoropyrimidines. Consistent with this, short-term exposure of bacterial strains to 5FU was sufficient to convert sensitive strains to resistant strains (Extended Data Fig. 2c).

The bioactivation of CAP to 5FU is generally considered to be uniquely catalyzed by mammalian enzymes expressed in the gastrointestinal tract and liver ${ }^{27}$. Surprisingly, we found that multiple gut bacteria were susceptible to the prodrug CAP (Fig. 1b) at physiological concentrations $(\sim 14 \mathrm{mg} / \mathrm{ml}$ following oral administration) ${ }^{28}$. With the exception of a single strain (Providencia rettgeri) we were able to determine a MIC, which spanned two orders of magnitude ( $\mathrm{min}=0.3 \mathrm{mg} / \mathrm{ml}$; $\max >10 \mathrm{mg} / \mathrm{ml}$ ) (Supplementary Table 1). Variation in sensitivity to CAP was significantly associated with 5FdUR sensitivity (rho=0.478, $p$-value=0.0007), but not 5FU (Extended Data Fig. 2d), suggesting that the ability for bacteria to import and/or activate this compound does not match the overall sensitivity to fluoropyrimidines.

Next, we sought to gain insight into the mechanism of action of fluoropyrimidines against human gut bacteria. We focused our initial efforts on Escherichia coli, due to its robust genetic tools ${ }^{29}$, high prevalence in the human gut $(>50 \% \text { of individuals })^{26}$, and intermediate level of sensitivity to $5 F U$ $(\mathrm{MIC}=62.5 \mu \mathrm{g} / \mathrm{ml}$ ), which would allow the identification of factors that increase resistance or sensitivity. 
In mammalian cells, the primary target of fluoropyrimidines is thymidylate synthase ${ }^{30}$, a key enzyme required for DNA, RNA, and protein biosynthesis across domains ${ }^{31}$. A key feature of this mechanism of action is that it is nutrient-dependent; excess uracil rescues drug sensitivity ${ }^{32}$. Bacterial sensitivity to $5 F U$, 5FdUR, and CAP was dependent on the culture media; E. coli assayed in rich culture media had a significantly higher MIC compared to minimal media (Fig. 2a). The sensitivity of E. coli to fluoropyrimidines was rescued by uracil in a dose-dependent manner (Fig. 2b); supplementation of 10 $\mu \mathrm{g} / \mathrm{ml}$ of uracil resulted in a 2500-, 40-, and 16-fold increase in MIC towards 5FU, 5FdUR, and CAP, respectively. These results suggest that uracil rescues growth due to countering the inhibition of pyrimidine metabolism by fluoropyrimidines.

Next, we used RNA-seq to gain a more comprehensive view of the metabolic pathways impacted by fluoropyrimidines. E. coli was grown to mid-exponential phase and then exposed to sub-MIC levels of CAP and 5FU (0.5X MIC in LB media) for $30 \mathrm{~min}(2.53 \pm 0.52$ million mRNA reads/sample; Supplementary Table 2). Both drugs has a marked impact on bacterial transcription relative to vehicle controls with 947 (5FU) and 663 (CAP) differentially expressed genes ${ }^{33}$ (fold-change $\geq|2|$, FDR<0.1; Extended Data Fig. 3a, b, Supplementary Table 3). This represented $21.8 \%$ (5FU) and $15.3 \%$ (CAP) of genes in the genome. While there was a significant correlation between fold-change in response to 5FU and CAP treatment $\left(R^{2}=0.163, p\right.$-value<0.0001), (Extended Data Fig. 3c) only $16.9 \%$ of the upregulated genes and $6.6 \%$ of the down-regulated genes were consistent between both drugs (Extended Data Fig. 3d).

Pathway enrichment analysis revealed that among other pathways pyrimidine metabolism was impacted by 5FU (Extended Data Fig. 3e), while flagellar assembly and primary metabolism pathway were impacted by CAP (Extended Data Fig. 3f). As seen for the overall transcriptional response, there was a significant correlation between fold-change in response to both drugs for the 46 genes involved in pyrimidine metabolism $\left(\mathrm{R}^{2}=0.199, p\right.$-value=0.0019; Fig. 2c, Supplementary Table 3$)$. To test if any of these genes play a causal role in bacterial sensitivity to fluoropyrimidines, we screened bacterial strains deficient for each of the 28 non-essential genes that were differentially expressed in response to either of the two drugs. Surprisingly, these results demonstrated that nearly all of these genes do not impact drug sensitivity, with the exception of upp (uracil phosphoribosyltransferase) (Supplementary Table 4). Consistent with prior studies ${ }^{34}$, upp-deficient $E$. coli displayed high-level resistance $(2,000$-fold increase in 5FU MIC) (Fig. 2d); however, this is the first report to our knowledge that demonstrates that an E. coli upp-deficient strain also display cross-resistance to CAP and 5FdUR (Fig. 2d). Targeted and whole genome sequencing of our 5FU-resistant mutants (Extended Data Fig. 2d) revealed multiple unique mutations within the upp genes of E. coli and B. fragilis (Supplementary Table 5). In strains with wildtype upp, we identified mutations within other pyrimidine metabolism genes, including uridine 
phosphorylase (E. coli), uridylate kinase (E. coli), and thymidine kinase (B. ovatus) (Supplementary Table 5). Taken together, our results support pyrimidine metabolism as a key target for fluoropyrimidines, while also demonstrating broader impacts of these compounds on pathways for primary metabolism, transport, and flagellar assembly (Extended Data Fig. 3e, 3f) that have yet to be directly implicated in drug sensitivity.

Research on antibiotics has demonstrated the importance of multiple resistance mechanisms, including target modification, bypass pathways, target overproduction, decreased cell penetrance, increased efflux, and enzymatic inactivation ${ }^{35,36}$. We reasoned that the latter might have the potential to impact treatment outcomes due to decreased drug bioavailability and/or gastrointestinal toxicity ${ }^{37}$. To test if drug resistant bacteria encode enzymes capable of drug inactivation (Fig. 3a), we screened the top 23 strains (49\% of the initial set), corresponding to a $5 \mathrm{FU}$ MIC cutoff of $50 \mu \mathrm{g} / \mathrm{ml}$, using a disk diffusion assay. This concentration is physiologically relevant as indicated by the peak plasma concentration of 5FU achieved during intravenous 5FU chemotherapy ${ }^{38}$. This bioassay screen (Fig. 3b, Extended Data Fig. 4a) identified two drug inactivating Proteobacteria: E. coli and Salmonella enterica. LC-QTOF/MS confirmed the depletion of 5FU with quantitative conversion to a single metabolite, dihydrofluorouracil (DHFU) (Fig. 3c). These results may help to explain why many of the tested E. coli pyrimidine metabolism mutants did not have increased sensitivity or resistance to $5 \mathrm{FU}$, due to the fact that $E$. coli converts nearly all of the administered drug to DHFU instead of all of the other theoretically possible downstream metabolites of 5FU (Extended Data Fig. 1). Consistent with this, attempts to detect and quantify peaks for other fluorinated compounds were unsuccessful (Supplementary Table 6); only 5FU and DHFU were detected.

In mammalian cells, dihydropyrimidine dehydrogenase (DPYD) is responsible for the biotransformation of $5 F U$ to DHFU ${ }^{39}$. Patients with DPYD deficiency are linked with high systemic exposure to $5 F U$ and adverse events ${ }^{39}$. In E. coli, DPYD is encoded by two neighboring genes: preT and preA found within the preTA operon ${ }^{40}$. Purified PreTA protein has been shown to be sufficient to catalyze the reduction of the pyrimidines uracil and thymine, as well as $5 \mathrm{FU}^{40}$; however, the genes necessary and sufficient for this activity in bacterial cells remained unknown. We generated a clean deletion of the preTA operon in E. coli MG1655 ( $\triangle$ preTA), which resulted in a complete loss-of-function in our bioassay (Extended Data Fig. 4b) and quantitatively confirmed by LC-QTOF/MS (Fig. 3d). Chromosomal complementation with a constitutively expressed preTA operon ( $\triangle$ preTA/pre $\left.T A^{+}\right)$resulted in an increased rate of metabolism relative to wild-type E. coli (Fig. 3d and Extended Data Fig. 4b). An empty vector control (E. coli $\triangle$ preTA/pINT1) had no impact on either assay. We confirmed the role of the preTA operon in 5FU inactivation with a second strain of E. coli (BW25113) (Fig. 3d, Extended Data Fig. 4c). Comparable growth was observed for each isogenic strain in the presence and absence of 5FU, with the 
exception of $\Delta$ preTA/preTA ${ }^{+}$, which showed a significant increase in drug tolerance in minimal media (Fig. 3e).

These results demonstrate that preTA is both necessary and sufficient for metabolism of 5FU by E. coli, and that high levels of preTA expression ameliorate the observed inhibition of bacterial growth. Consistent with these results, E. coli MG1655 growth was unaffected by DHFU (MIC>1 mg/ml). We reasoned that the bacterial biotransformation of 5FU by preTA would similarly interfere with the activity of this drug against cancer cells. Cell-free conditioned media from each of our engineered $E$. coli strains was added to the colorectal cancer cell line HCT-116. Uninoculated media and preTA-deficient conditioned media supplemented with 5FU inhibited cancer cell proliferation; however, this effect was fully rescued by wild-type $E$. coli and the $\triangle$ preTA/preTA ${ }^{+}$strains (Fig. 3f).

Given the conservation between mammalian DPYD and bacterial PreTA we were surprised that only two strains were identified in our screen for 5FU inactivating bacteria. Consistent with our bioassay results, a tBLASTn search querying PreTA against the draft genomes of all 22 of the tested strains only revealed a single pair of genomic loci from S. enterica (84\% and $94 \%$ full length amino acid identity to PreT and PreA, respectively). To identify other putative metabolizing strains, we built a profile Hidden Markov Model (HMM) and used it to search for orthologs of pre $T$ and pre $A$ across 9,082 bacterial isolate genomes. To minimize false positives due to the fact that both PreT and PreA are part of large protein families with a range of functions and substrate specificities ${ }^{41}$, we required that the pre $T$ and pre $A$ orthologs were adjacent in the genome and on the same strand. This analysis revealed 1704 putative preTA operons from 407 species, mainly in Proteobacteria (1509 operons from 292 species) and Firmicutes (172 operons from 92 species) (Fig. 4, Supplementary Table 7). This phylum-level distribution was similar for species with an estimated gut prevalence over $5 \%(n=23)$ and for species with a gut prevalence of $1 \%$ or lower $(n=109)^{42}$. As expected, preTA was conserved in close relatives of $E$. coli, including other Escherichia, Salmonella, and Citrobacter. However, we also found operons in more distantly related Betaproteobacteria (e.g., Oxalobacter formigenes) and diverse Firmicutes: Anaerostipes hadrus, Eubacterium hallii, and Lactobacillus reuteri. These results were consistent in an independent analysis of 55,427 metagenome-assembled genomes (MAGs) ${ }^{43}$, resulting in the identification of 74 additional preTA operons, all from human gut MAGs, $89 \%$ of which were Firmicutes or Proteobacteria (Extended Data Fig. 5, Supplementary Table 8).

We validated five putative preTA orthologs through a combination of whole cell assays and heterologous expression. Incubation of preTA-positive strains confirmed the ability of the Firmicutes species Anaerostipes caccae and Clostridium sporogenes to metabolize 5FU (Fig. 4, Extended Data Fig. 6). Heterologous expression in the E. coli $\triangle$ preTA strain enabled us to validate preTA orthologs detected in the Gammaproteobacterium S. enterica, the Betaproteobacterium O. formigenes, and the 
Firmicute Lactobacillus reuteri (Fig. 4, Extended Data Fig. 6). Together, these results demonstrate that bacterial PreTA can maintain its activity against 5FU despite considerable phylogenetic and primary sequence divergence (66-93\% similarity). Finally, we determined the prevalence of each preTA encoding species in the human gut. This analysis identified 4 highly prevalent preTA encoding bacterial species (>90\% of the analyzed samples), including the Firmicute $A$. hadrus and the Proteobacterium E. coli (Fig. 4, Supplementary Table 7). An additional 13 isolate genomes and 8 MAGs were identified in $>10 \%$ of the analyzed samples (Fig. 4, Extended Data Fig. 6, Supplementary Table 7, 8). Of note, bacteria that carry the preTA operon have been detected in tumor samples across multiple cancer types ${ }^{5,44-47}$, suggesting that this enzyme may be relevant outside of the gastrointestinal tract.

In conclusion, our results demonstrate how pathways for sensitivity to and metabolism of fluoropyrimidine drugs are conserved across two domains of life. The implications of this are many, suggesting that the mechanistic dissection of drug mechanism of action in bacteria may provide translational insights for host tissues and prompting a broader view of the off-target effects (i.e. toxicity) of therapeutics and the degree to which drug-induced shifts in microbial community structure and function have downstream consequences for drug efficacy or toxicity. Importantly, although our in vitro assays suggest that bacterial drug inactivation in the gastrointestinal tract or even within diseased tissue ${ }^{5}$ could interfere with drug efficacy, more work is needed to assess the relative impact of this biotransformation on efficacy versus the dose-limiting gastrointestinal side effects, as previously demonstrated for the anticancer drug irinotecan ${ }^{8}$. These results are also consistent with another recent study ${ }^{9}$, which highlighted the unexpected overlap between host and bacterial drug metabolites. Traditional approaches for studying drug disposition do not distinguish these two alternatives ${ }^{37}$, which may explain the difficulties in predicting fluoropyrimidine toxicity using only human genotypic information. Furthermore, our discovery of diverse preTA positive strains could open the door towards preventing the severe, and at times lethal, toxicity observed from patients undergoing fluoropyrimidine chemotherapy with loss-of-function mutations in mammalian DPYD 39 . 


\section{REFERENCES}

1. Falony, G. et al. Population-level analysis of gut microbiome variation. Science 352, 560-564 (2016).

2. Maier, L. et al. Extensive impact of non-antibiotic drugs on human gut bacteria. Nature 555, 623628 (2018).

3. Chankhamjon, P. et al. Systematic mapping of drug metabolism by the human gut microbiome. (2019). doi:10.1101/538215

4. Zimmermann, M., Zimmermann-Kogadeeva, M., Wegmann, R. \& Goodman, A. L. Mapping human microbiome drug metabolism by gut bacteria and their genes. Nature (2019). doi:10.1038/s41586019-1291-3

5. Geller, L. T. et al. Potential role of intratumor bacteria in mediating tumor resistance to the chemotherapeutic drug gemcitabine. Science 357, 1156-1160 (2017).

6. Scott, T. A. et al. Host-Microbe Co-metabolism Dictates Cancer Drug Efficacy in C. elegans. Cell 169, 442-456.e18 (2017).

7. Wallace, B. D. et al. Structure and Inhibition of Microbiome $\beta$-Glucuronidases Essential to the Alleviation of Cancer Drug Toxicity. Chem. Biol. 22, 1238-1249 (2015).

8. Wallace, B. D. et al. Alleviating cancer drug toxicity by inhibiting a bacterial enzyme. Science $\mathbf{3 3 0}$, 831-835 (2010).

9. Zimmermann, M., Zimmermann-Kogadeeva, M., Wegmann, R. \& Goodman, A. L. Separating host and microbiome contributions to drug pharmacokinetics and toxicity. Science 363 , (2019).

10. Arruebo, M. et al. Assessment of the evolution of cancer treatment therapies. Cancers 3, 32793330 (2011).

11. Gadiko, C. et al. Comparative bioavailability study of capecitabine tablets of $500 \mathrm{mg}$ in metastatic breast cancer and colorectal cancer patients under fed condition. Clin. Res. Regul. Aff. 29, 72-76 (2012). 
12. Haller, D. G. et al. Potential regional differences for the tolerability profiles of fluoropyrimidines. J. Clin. Oncol. 26, 2118-2123 (2008).

13. Jennings, B. A. et al. Evaluating Predictive Pharmacogenetic Signatures of Adverse Events in Colorectal Cancer Patients Treated with Fluoropyrimidines. PLoS ONE 8, e78053 (2013).

14. Saif, M. W., Syrigos, K., Mehra, R., Mattison, L. K. \& Diasio, R. B. Dihydropyrimidine dehydrogenase deficiency (DPD) in GI malignancies: experience of 4-years. Pak. J. Med. Sci. Q. 23, 832-839 (2007).

15. Leonard, R., Hennessy, B. T., Blum, J. L. \& O'Shaughnessy, J. Dose-adjusting capecitabine minimizes adverse effects while maintaining efficacy: a retrospective review of capecitabine for metastatic breast cancer. Clin. Breast Cancer 11, 349-356 (2011).

16. Horowitz, J., Saukkonen, J. J. \& Chargaff, E. Effects of fluoropyrimidines on the synthesis of bacterial proteins and nucleic acids. J. Biol. Chem. 235, 3266-3272 (1960).

17. Bloch, A. \& Hutchison, D. J. A Mechanisms of resistance to fluoropyrimidines. Cancer Res. 24, 433-439 (1964).

18. Stringer, A. M. et al. Gastrointestinal microflora and mucins may play a critical role in the development of 5-Fluorouracil-induced gastrointestinal mucositis. Exp. Biol. Med. 234, 430-441 (2009).

19. Von Bültzingslöwen, I., Adlerberth, I., Wold, A. E., Dahlén, G. \& Jontell, M. Oral and intestinal microflora in 5-fluorouracil treated rats, translocation to cervical and mesenteric lymph nodes and effects of probiotic bacteria. Oral Microbiol. Immunol. 18, 278-284 (2003).

20. Stringer, A. M. et al. Chemotherapy-induced diarrhea is associated with changes in the luminal environment in the DA rat. Exp. Biol. Med. 232, 96-106 (2007).

21. Zwielehner, J. et al. Changes in human fecal microbiota due to chemotherapy analyzed by TaqMan-PCR, 454 sequencing and PCR-DGGE fingerprinting. PLoS One 6, e28654 (2011).

22. van Vliet, M. J. et al. Chemotherapy treatment in pediatric patients with acute myeloid leukemia receiving antimicrobial prophylaxis leads to a relative increase of colonization with potentially 
pathogenic bacteria in the gut. Clin. Infect. Dis. 49, 262-270 (2009).

23. Stringer, A. M. et al. Biomarkers of chemotherapy-induced diarrhoea: a clinical study of intestinal microbiome alterations, inflammation and circulating matrix metalloproteinases. Support. Care Cancer 21, 1843-1852 (2013).

24. Montassier, E. et al. Chemotherapy-driven dysbiosis in the intestinal microbiome. Aliment. Pharmacol. Ther. 42, 515-528 (2015).

25. García-González, A. P. et al. Bacterial Metabolism Affects the C. elegans Response to Cancer Chemotherapeutics. Cell 169, 431-441.e8 (2017).

26. Nayfach, S., Fischbach, M. A. \& Pollard, K. S. MetaQuery: a web server for rapid annotation and quantitative analysis of specific genes in the human gut microbiome. Bioinformatics 31, 3368-3370 (2015).

27. Walko, C. M. \& Lindley, C. Capecitabine: a review. Clin. Ther. 27, 23-44 (2005).

28. Zhang, L., Zhang, Y. D., Strong, J. M., Reynolds, K. S. \& Huang, S.-M. A regulatory viewpoint on transporter-based drug interactions. Xenobiotica 38, 709-724 (2008).

29. Baba, T. et al. Construction of Escherichia coli K-12 in-frame, single-gene knockout mutants: the Keio collection. Mol. Syst. Biol. 2, (2006).

30. Longley, D. B., Harkin, D. P. \& Johnston, P. G. 5-fluorouracil: mechanisms of action and clinical strategies. Nat. Rev. Cancer 3, 330-338 (2003).

31. Islam, Z. et al. Bacterial versus human thymidylate synthase: Kinetics and functionality. PLoS One 13, e0196506 (2018).

32. Pinedo, H. M. \& Peters, G. F. Fluorouracil: biochemistry and pharmacology. J. Clin. Oncol. 6, 1653-1664 (1988).

33. Love, M. I., Huber, W. \& Anders, S. Moderated estimation of fold change and dispersion for RNAseq data with DESeq2. Genome Biol. 15, 550 (2014).

34. O’Donovan, G. A. \& Neuhard, J. Pyrimidine metabolism in microorganisms. Bacteriol. Rev. 34, 278-343 (1970). 
35. Lewis, K. Platforms for antibiotic discovery. Nat. Rev. Drug Discov. 12, 371-387 (2013).

36. Spanogiannopoulos, P., Waglechner, N., Koteva, K. \& Wright, G. D. A rifamycin inactivating phosphotransferase family shared by environmental and pathogenic bacteria. Proc. Natl. Acad. Sci. U. S. A. 111, 7102-7107 (2014).

37. Bisanz, J. E., Spanogiannopoulos, P., Pieper, L. M., Bustion, A. E. \& Turnbaugh, P. J. How to Determine the Role of the Microbiome in Drug Disposition. Drug Metab. Dispos. 46, 1588-1595 (2018).

38. Casale, F. et al. Plasma concentrations of 5-fluorouracil and its metabolites in colon cancer patients. Pharmacol. Res. 50, 173-179 (2004).

39. Lunenburg, C. A. T. C. et al. Prospective DPYD genotyping to reduce the risk of fluoropyrimidineinduced severe toxicity: Ready for prime time. Eur. J. Cancer 54, 40-48 (2016).

40. Hidese, R., Mihara, H., Kurihara, T. \& Esaki, N. Escherichia coli dihydropyrimidine dehydrogenase is a novel NAD-dependent heterotetramer essential for the production of 5,6-dihydrouracil. J. Bacteriol. 193, 989-993 (2011).

41. Barba, M., Dutoit, R., Legrain, C. \& Labedan, B. Identifying reaction modules in metabolic pathways: bioinformatic deduction and experimental validation of a new putative route in purine catabolism. BMC Syst. Biol. 7, 99 (2013).

42. Bradley, P. H., Nayfach, S. \& Pollard, K. S. Phylogeny-corrected identification of microbial gene families relevant to human gut colonization. PLoS Comput. Biol. 14, e1006242 (2018).

43. Nayfach, S., Shi, Z. J., Seshadri, R., Pollard, K. S. \& Kyrpides, N. C. New insights from uncultivated genomes of the global human gut microbiome. Nature 568, 505-510 (2019).

44. Nakatsu, G. et al. Gut mucosal microbiome across stages of colorectal carcinogenesis. Nat. Commun. 6, 8727 (2015).

45. Lu, Y. et al. Mucosal adherent bacterial dysbiosis in patients with colorectal adenomas. Sci. Rep. 6, 26337 (2016).

46. Urbaniak, C. et al. The Microbiota of Breast Tissue and Its Association with Breast Cancer. Appl. 
Environ. Microbiol. 82, 5039-5048 (2016).

47. Banerjee, S. et al. The ovarian cancer oncobiome. Oncotarget 8, 36225-36245 (2017).

48. Guindon, S., Delsuc, F., Dufayard, J.-F. \& Gascuel, O. Estimating maximum likelihood phylogenies with PhyML. Methods Mol. Biol. 537, 113-137 (2009).

49. Edgar, R. C. MUSCLE: multiple sequence alignment with high accuracy and high throughput. Nucleic Acids Res. 32, 1792-1797 (2004).

Correspondence and requests for materials should be addressed to Peter J. Turnbaugh (peter.turnbaugh@ucsf.edu).

\section{Acknowledgements}

We are indebted to the other members of the Gerona, Pollard, and Turnbaugh labs for their helpful suggestions during the preparation of this manuscript. We thank Brian Yu, Michelle Tan, and Rene Sit from the Chan-Zuckerberg Biohub for assistance with DNA sequencing; Gerry Wright and Linda Ejim for providing pINT1; and Jordan Bisanz for advice with computational methods. This work was supported by the National Institutes of Health (R01HL122593; R21CA227232) and the Searle Scholars Program (SSP2016-1352). P.J.T. is a Chan Zuckerberg Biohub investigator and a Nadia's Gift Foundation Innovator supported, in part, by the Damon Runyon Cancer Research Foundation (DRR-42-16). Fellowship support was provided by the Canadian Institutes of Health Research (P.S. and K.N.L.).

\section{Author contributions}

P.S. performed and supervised all of the experimental work. P.H.B. performed the bioinformatic analysis of preTA operons across bacteria and human metagenomes. J.M. and Y.N.A.M. developed and performed the mass spectrometry quantification of $5 F U$ and DHFU from conditioned media samples. 
bioRxiv preprint doi: https://doi.org/10.1101/820084; this version posted October 25,2019 . The copyright holder for this preprint (which was not certified by peer review) is the author/funder, who has granted bioRxiv a license to display the preprint in perpetuity. It is made available under aCC-BY-ND 4.0 International license.

K.N.L. performed the sequencing and analysis of 5FU-resistant mutants. P.J.T., R.R.G., and K.S.P. supervised the study. P.S. and P.J.T. wrote the manuscript with input from all authors.

\section{Competing interests}

P.J.T. is on the scientific advisory boards for Kaleido, Pendulum, Seres, and SNIPRbiome; there is no direct overlap between the current study and these consulting duties. All other authors have no relevant declarations. 


\section{FIGURE LEGENDS}

a

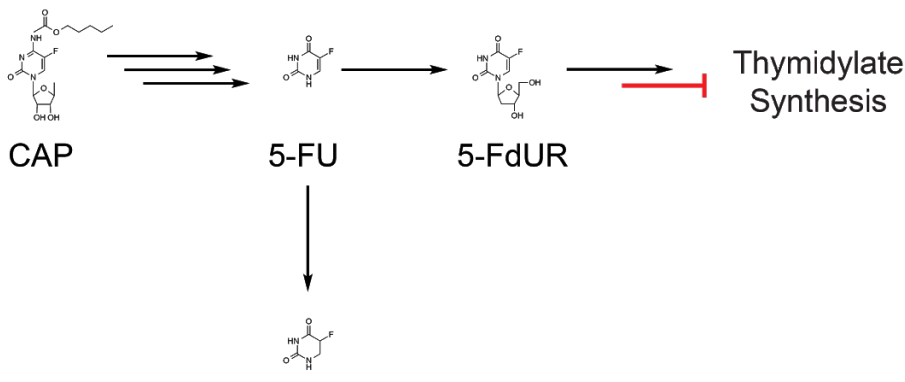

DHFU

b

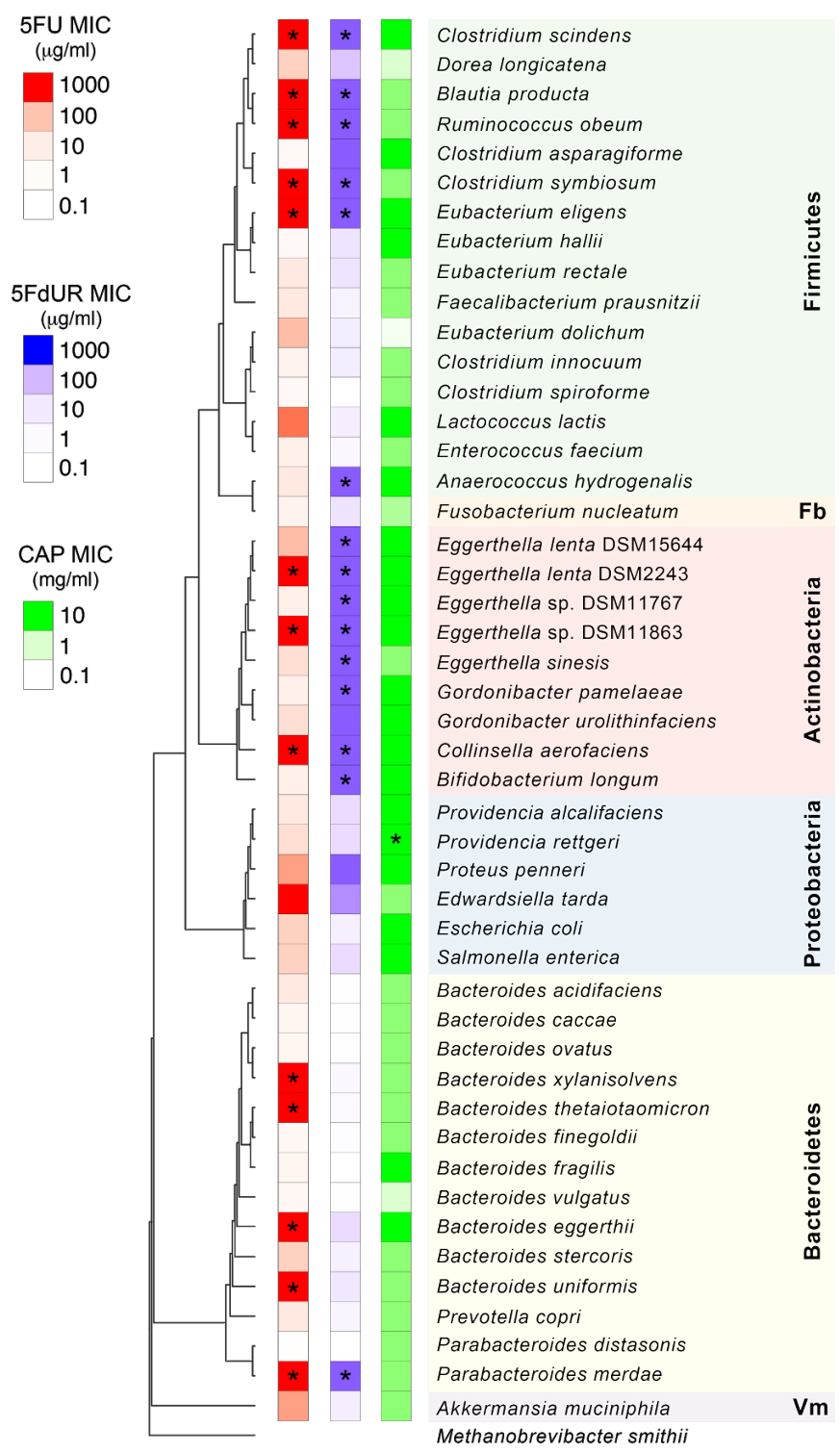

Figure 1. Fluoropyrimidines inhibit the growth of gut bacteria from multiple phyla (a) Simplified metabolic pathways for the clinically used fluoropyrimidines. (b) Susceptibility of gut bacteria to fluoropyrimidines. Values represent the minimal inhibitory concentration (MIC, $<10 \%$ growth relative to vehicle controls) of each drug during the growth of each of 47 representative human gut bacterial isolates in mono-culture

$(n=2$ replicates/strain/concentration). Asterisks represent strains where no MIC was achieved: $>1000 \mu \mathrm{g} / \mathrm{ml}$ (5FU), $>500 \mu \mathrm{g} / \mathrm{ml}$ (5FdUR), and $>10 \mathrm{mg} / \mathrm{ml}$ (CAP). The phylogenetic tree was built using PhyML ${ }^{48}$, based on a multiple alignment made using MUSCLE ${ }^{49}$ of the full-length 16S rRNA genes for each strain. Fb, Fusobacteria; Vm, Verrucomicrobia. 
a
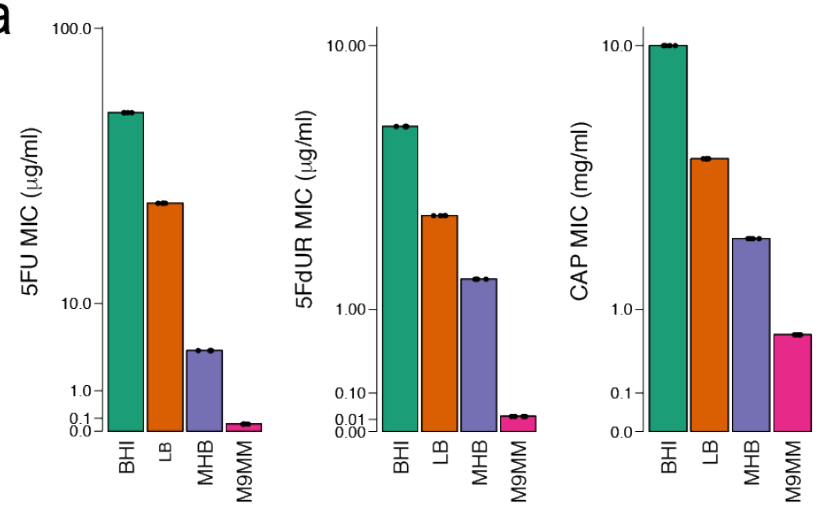

C

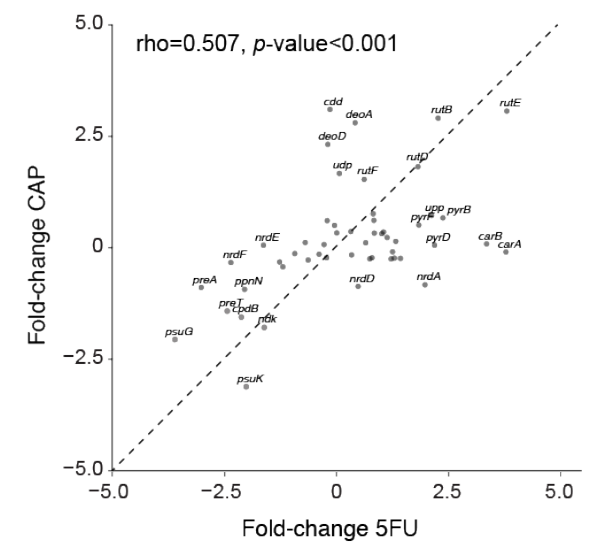

b
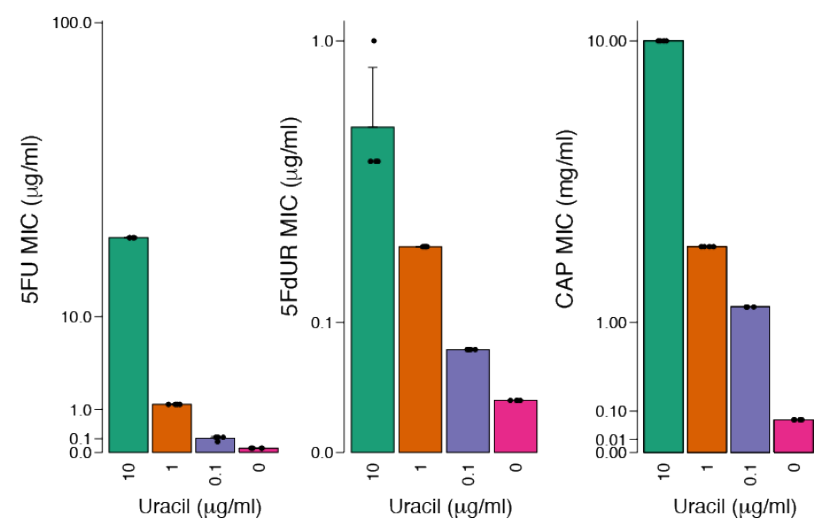

d
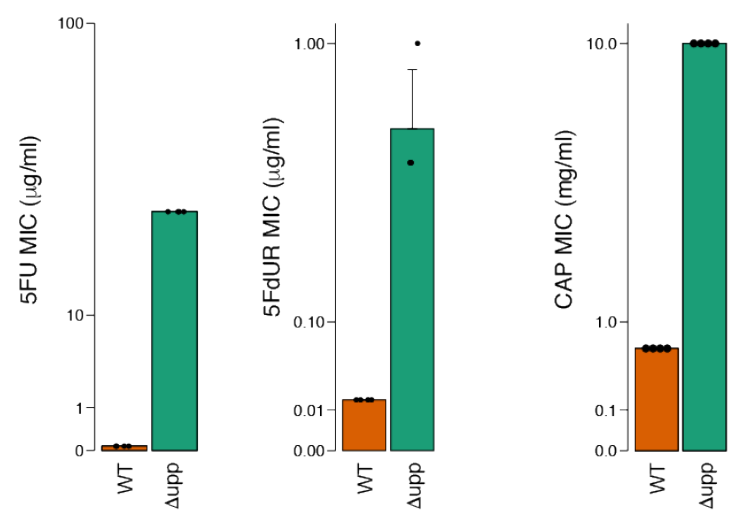

Figure 2. Fluoropyrimidines target bacterial pyrimidine metabolism. (a) Rich media rescues the impact of 5FU and CAP on the growth of E. coli BW25113. Media used: BHI (Brain heart infusion broth); LB (lysogeny broth); MHB (Mueller Hinton II broth, cation adjusted); and M9MM (M9 minimal salts media plus glucose). (b) Uracil rescues the growth of E. coli BW25113 in the presence of 5FU, 5FdUR, and CAP in a dose dependent-manner. M9MM plus glucose was used as the background with added uracil from $0.1-10 \mu \mathrm{g} / \mathrm{ml}$. (c) Correlation between the differential expression of genes involved in pyrimidine metabolism ( $n=46)$ in response to $5 F U$ versus CAP. Dotted lines depict $\log _{2}$ fold changes of -1 and 1 . (d) A loss-of-function mutation in uracil phosphoribosyltransferase gene $(\triangle u p p)$ rescues the growth of $E$. coli BW25113 in the presence of 5FU and CAP. MIC assay performed in M9MM plus glucose. Error bars represent standard deviation. 
a

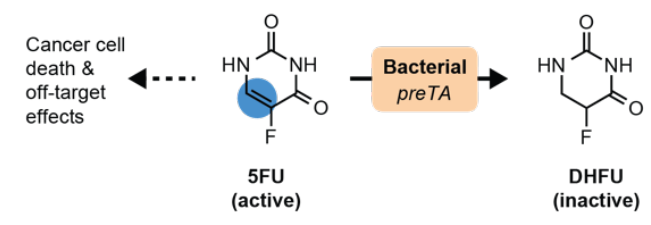

b

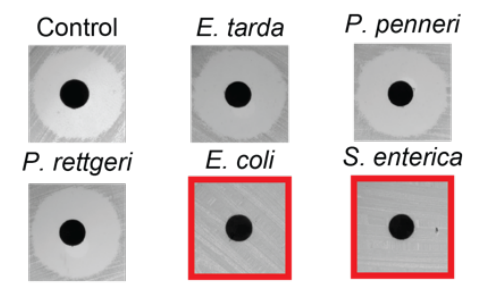

C

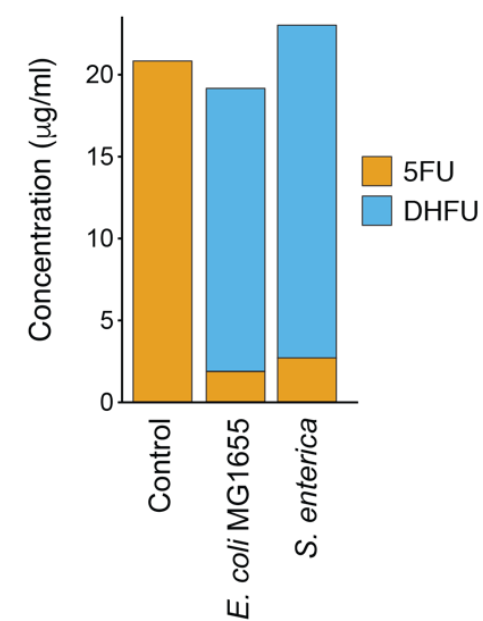

d

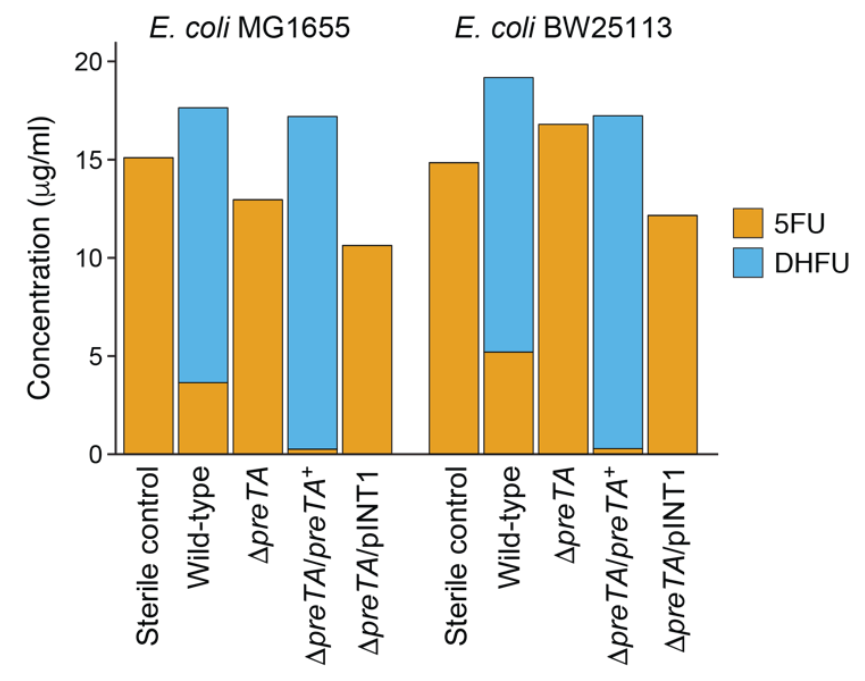

e

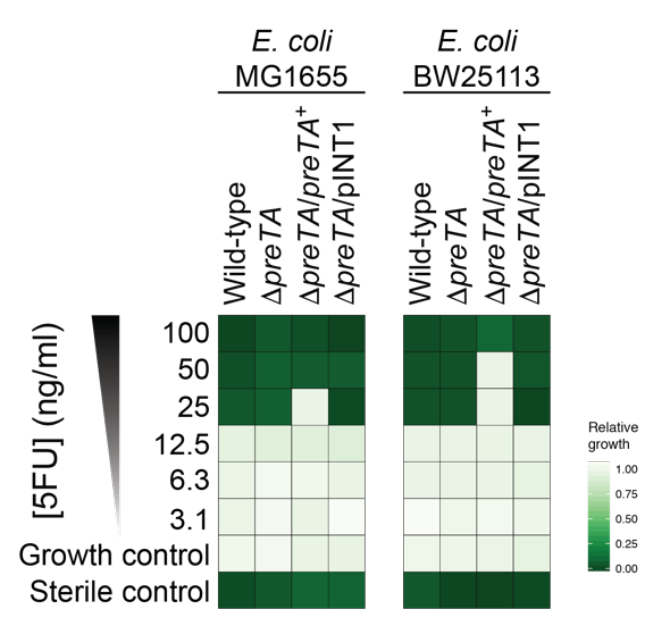

$f$

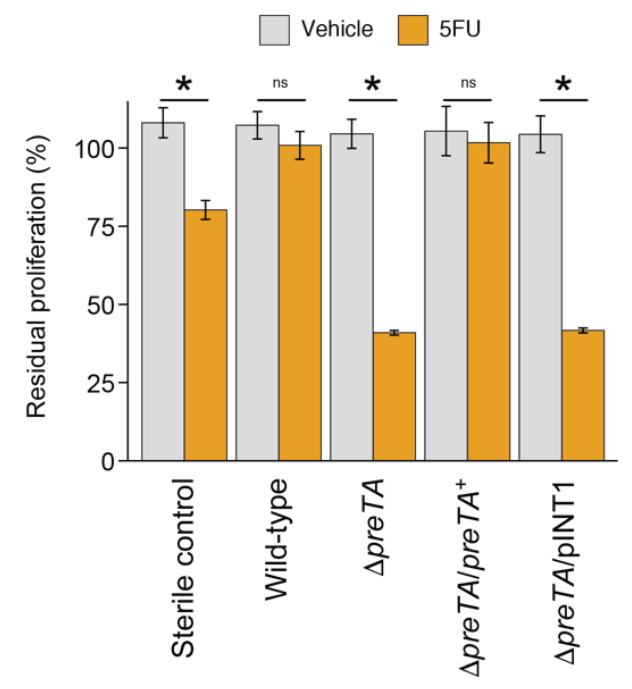

Figure 3. Drug resistant bacteria inactivate 5FU through reduction to DHFU. (a) Canonical pathway for the elimination of 5FU in hepatocytes. The host enzyme and bacterial homolog (described here) are labeled. (b) $235 \mathrm{FU}$-resistant gut bacteria (MIC>50 $\mu \mathrm{g} / \mathrm{ml}$ ) were incubated in $\mathrm{BHI}^{+}$with $20 \mu \mathrm{g} / \mathrm{ml}$ of $5 \mathrm{FU}$. 5 select strains with the sterile control are shown here with the remaining strains provided in Extended Data Fig. 4. Conditioned media was assayed using a disk diffusion test. The two identified 5FU 
inactivating strains are highlighted in red. (c) Confirmation of 5FU metabolism to DHFU by LC-QTOF/MS. Sterile controls and conditioned media of E. coli MG1655 and S. enterica DSM17058 following 48 hours anaerobic incubation with $20 \mu \mathrm{g} / \mathrm{ml}$ of $5 \mathrm{FU}$ were analyzed. (d) Isogenic $E$. coli strains were incubated with 5FU and conditioned media was sampled and assayed for residual 5FU concentration by LCQTOF/MS (shown here, 48 hours after inoculation) and disk diffusion (shown in Extended Data Fig. 4). (e) 5FU MIC determination of $E$. coli strains constructed with various preTA states in rich $(\mathrm{BHI})$ and minimal (M9MM) media. (f) Conditioned media samples from the same panel of $E$. coli strains incubated for 72 hours in the presence or absence of 5FU were added to the colorectal cell line HCT-116 and cell proliferation was quantified using the MTT assay ( $n=3$ biological replicates/strain/condition). Error bars represent standard deviation. ${ }^{* * *} p$-value $<0.001$, Student's $t$ test. 


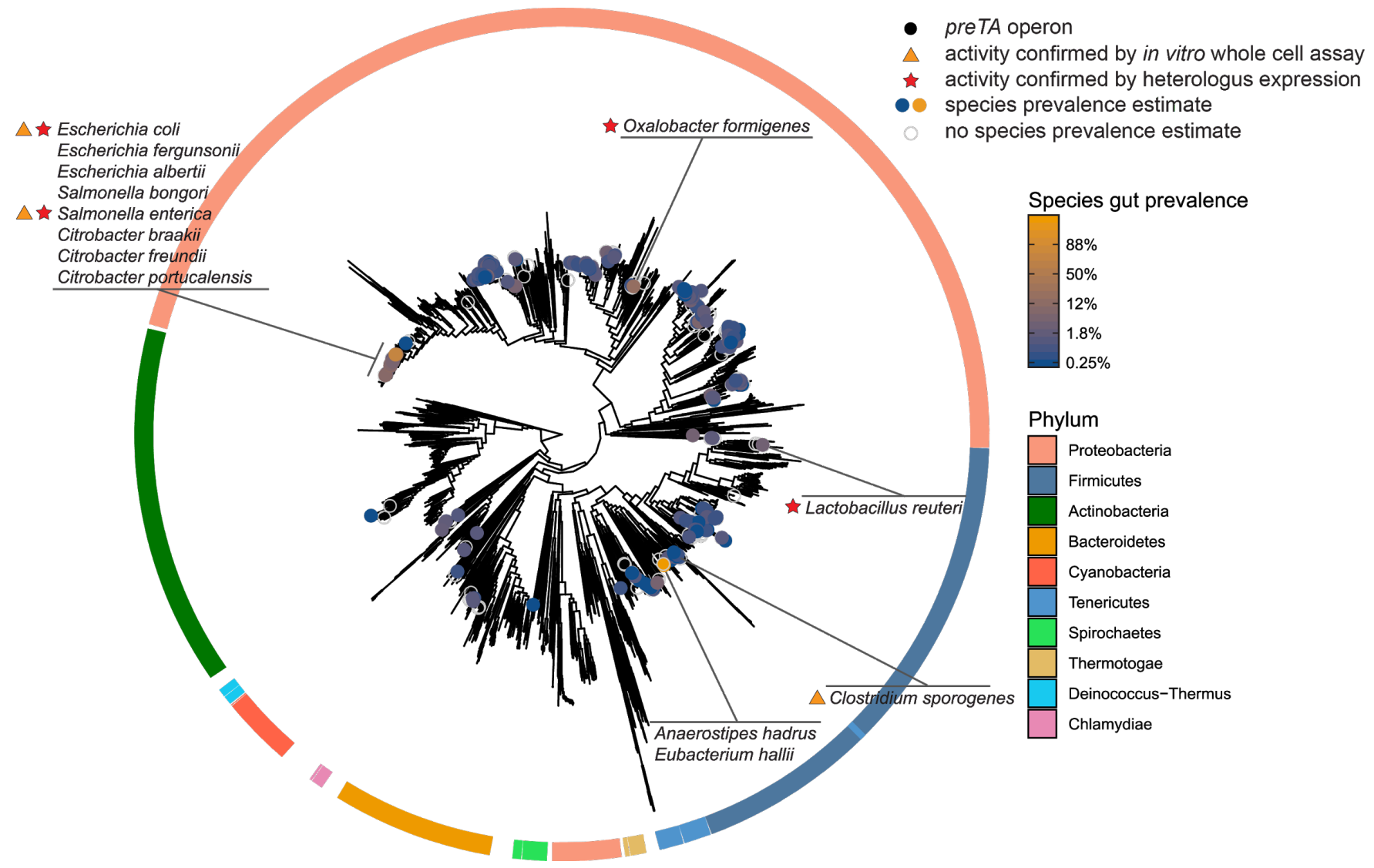

Figure 4. Functional orthologs of the preTA operon are widespread in human gut bacterial strains from the Firmicutes and Proteobacteria phyla. Distribution of bioinformatically-identified preTA operons across RefSeq bacterial genomes. A phylogenetic tree of these genomes made using a concatenated alignment of single-copy marker genes is shown. Bacterial species identified as carriers of putative preTA operons are identified with colored circles, where the color of the circle corresponds to prevalence levels from human gut microbiomes (blue: low prevalence; orange: high prevalence; unfilled grey: no prevalence estimate). Phylum-level annotations are shown as colored ring segments surrounding the tree for the ten phyla with the most species in Refseq. Specific taxa of interest are highlighted in call-out boxes. Red stars indicate preTA operons that have been validated to inactive 5FU by heterologous expression from $E$. coli $\triangle$ preTA (see Extended Data Fig. 6a). Orange triangles indicate preTA-positive bacterial species for which we have confirmed 5FU inactivation in vitro (see Extended Data Fig. 6b). A list of preTA-positive bacteria can be found in Supplementary Table 7. 